\title{
NEW PHARMACOLOGICAL TREATMENTS FOR PATIENTS WITH CHRONIC OBSTRUCTIVE PUMONARY DISEASE (COPD)
}

\author{
Nancy Elizabeth Larocca ${ }^{a}$, Dolores Moreno ${ }^{a}$, Jenny Valentina Garmendia ${ }^{\mathrm{b}}$, \\ Juan Bautista De Sanctis ${ }^{\mathrm{b} *}$
}

\author{
a Department of Pathology and Physiopathology, Experimental Medicine Institute, Faculty of Medicine, Universidad Central \\ de Venezuela \\ ${ }^{b}$ Departments of Clinical and Basic Immunology, Institute of Immunology, Faculty of Medicine, Universidad Central de \\ Venezuela \\ E-mail: sanctisj@gmail.com
}

Received: October 2, 2010; Accepted: February 8, 2011

Key words: COPD/Pharmacological treatment/B agonists/Muscarinic receptor antagonists/Phosphodiesterase inhibitors/ NKkb inhibitors/PI3K inhibitors

Background. Chronic Obstructive Pulmonary Disease (COPD) is a preventable and treatable disease characterized mainly by pulmonary airflow limitation that is not fully reversible. The airflow limitation is usually progressive and associated with abnormal inflammatory response of the lung to noxious particles or gasses. New different pharmacological approaches to decrease inflammation of the airways and consequently disease progression and increase airway obstruction reversibility have been developed.

Methods and Results. A literature search using PubMed, Science Direct, EBSCO and free patents on line for the years 2000-2010.

Conclusions. Recent discoveries in the physiology and pathology of airways diseases have served to generate potential new drugs for the treatment of COPD patients. Several substances that block or activate specific pathways and receptors the aim of which is to decrease inflammation and increase airway obstruction reversibility are being used in different clinical protocols and hopefully will be available for patients in the near future.

\section{INTRODUCTION}

Chronic Obstructive Pulmonary Disease (COPD) is a preventable and treatable disease with some significant extrapulmonary effects that may contribute to the severity in individual patients. Its pulmonary component is characterized by airflow limitation that is not fully reversible. The airflow limitation is usually progressive and associated with abnormal inflammatory response of the lung to noxious particles or gasses. It is observed mostly in elderly patients ( $>60$ years), (American Thoracic Society(ATS)/ European Respiratory Society (ERS) along with the Global Initiative for Obstructive Lung Disease 2009 $(\text { GOLD })^{1-3}$ COPD has become a major global epidemic which is increasing throughout the world. The general prevalence is $10 \%$; however, in most developing countries the incidence is higher ${ }^{4-6}$. Due to the increasing number of patients, it is expected that COPD will be the third leading cause of death worldwide by 2020 (ref. ${ }^{5,6}$ ).

COPD is characterized by two main pathophysiologic features: the development of inflammation of the small airways and obstruction, and emphysema. The airflow limitation in COPD is usually progressive and it is associated with an abnormal inflammatory response to different stimuli including tobacco use, pollution and pulmonary infection ${ }^{1-3}$. Mucus hypersecretion and cillary dysfunction lead to the collapse of damaged small airways producing airflow limitation, gas trapping and the characteristic ob- structive picture observed in spirometry test ${ }^{1-3}$. Patients with frequent exacerbations may have a more rapid decline in lung function and a reduced quality of life. In addition, patients with moderate to severe disease, who continue to smoke and are not receiving appropriate therapy, exhibit a median decline in forced expiratory volume in 1 second $\left(\mathrm{FEV}_{1}\right)$ of about $40-60 \mathrm{ml} / \mathrm{year}^{1-5}$. While the aetiology of COPD is certainly associated with smoking (around $80 \%$ ), the phenotype observed in COPD is variable and complex, and is likely the result of both various genetic and environmental factors ${ }^{1-5}$.

Around $50-70 \%$ of the exacerbations in COPD are associated with infection by bacteria and viruses ${ }^{1-6}$. The most frequent infectious agents isolated are Haemophilus influenzae, Streptococcus pneumoniae, Moraxella catarrhalis, Mycoplasma pneumoniae, Chlamydophila pneumoniae, rhinovirus, adenovirus, and respiratory syncytial virus ${ }^{6}$. When exacerbations occur, there is an increased secretion of chemokines (ENA-78 and IL-8), TNF- $\alpha$, and C-reactive protein that induce an increase number of neutrophils in the airways and consequently the amplification of the inflammatory response. In contrast, in the airways of stable COPD patients, the main cell populations are macrophages and CD8+ T lymphocytes ${ }^{4,5}$.

For COPD patients there is no therapeutic strategy available that induces a sustained improvement of lung function decline, with one exception: smoking cessation in early stages of the disease ${ }^{1,7-11}$. There are several reasons 
why drug development in COPD has proved to be very difficult: 1) there is little information about biomarkers (blood, sputum or breath), to monitor the short-term efficacy and predict the long-term potential of new treatments, 2) the animal models of COPD have not been useful, and 3 ) the high incidence co-morbidities of elderly populations, such as hypertension, ischaemic heart disease and diabetes ${ }^{10,11}$. However, recent research on cellular and molecular mechanisms COPD has promoted the increased interest of researchers and the pharmaceutical industry and novel approaches have been proposed ${ }^{9-13}$.

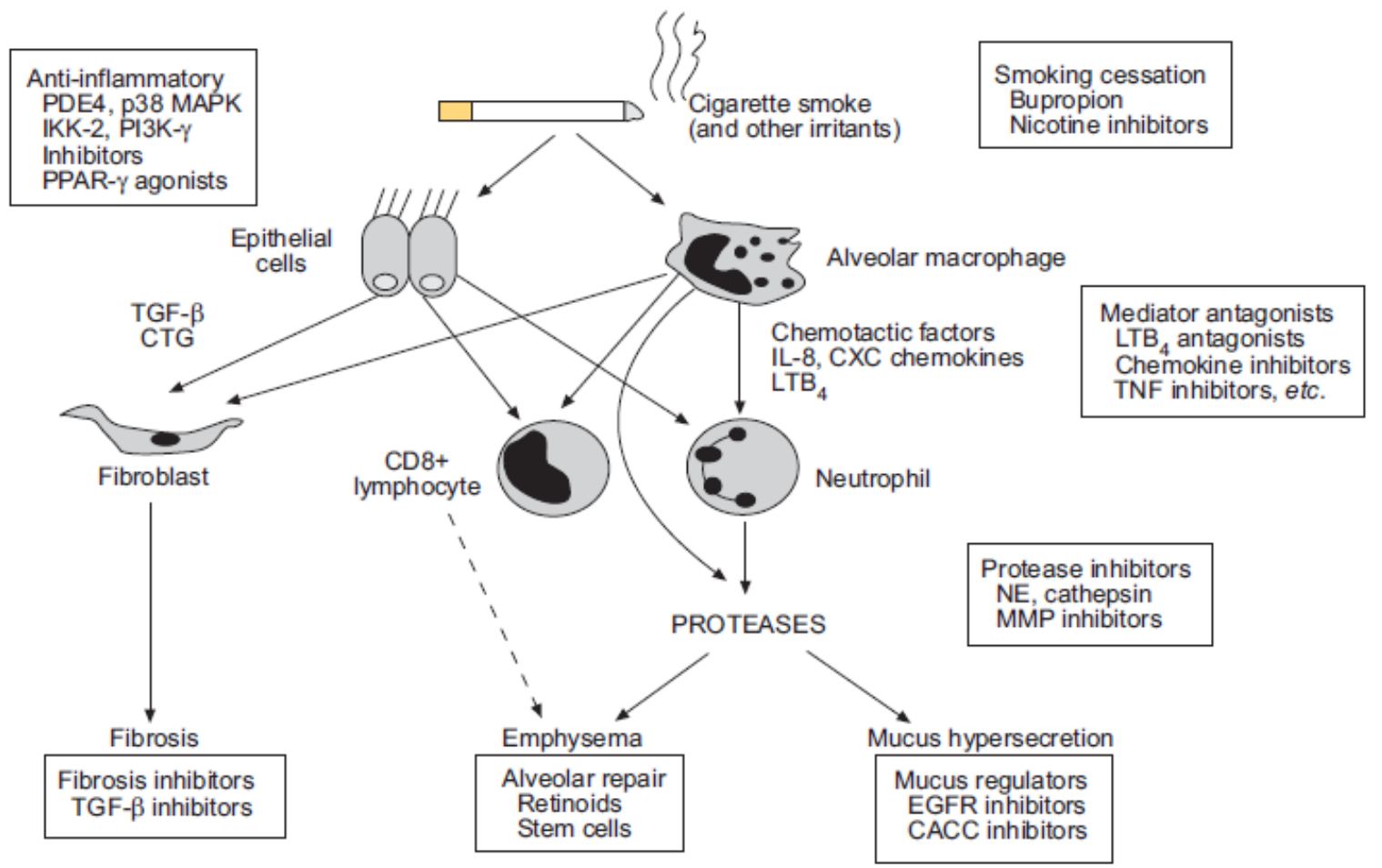

Fig. 1. The figure represents the different pharmacological strategies for COPD treatment. In a box, a general approach is illustrated in a schematic representation. Adapted from ${ }^{13}$.

\section{NEW DRUG DEVELOPMENTS IN COPD}

Treatment of patients with COPD usually follows the current guidelines of the GOLD Initiative (Global Strategy for the Diagnosis, Management, and Prevention of COPD). Patients are classified by FEV1 and FEV1/ forced vital capacity into different stages of severity (I - IV). According to this classification, patients should be treated with short-acting bronchodilators in all stages and one or more long-acting bronchodilators should be added from moderate (II) up to very severe (IV) stages. If patients have repeated exacerbations, inhaled glucocorticosteroids should be added in severe (III) and very severe (IV) $\mathrm{COPD}^{1,8}$. Thus, bronchodilators are the first-line therapy for COPD, either short acting ( $4 \mathrm{~h}$ duration) or long acting (12-24 h duration). There are two main types of bronchodilators in clinical use: a) $\beta$ 2-receptor agonists, and b) muscarinic (M3) acetylcholine (Ach) receptor antagonists. Both drug classes lead to airway smooth muscle relaxation. They provide effective symptomatic relief that is related to reduce dynamic hyperinflation by increasing airway diameters. Dynamic hyperinflation results from trapped air that is due to differences in the volume of air moved during inhalation and exhalation and is particularly relevant during exercise ${ }^{1,8}$. Both $\beta 2$ agonist and anticholinergic therapy act mainly by inducing bron- chodilatation, thereby reducing dyspnea and exacerbation frequency ${ }^{1,12,13}$.

Both inhaled Long Acting $\beta 2$ agonists (LABAs) and Short Acting $\beta 2$ agonists (SABAs) are used in the symptomatic treatment of $\mathrm{COPD}^{12,13}$. There are at present two LABA in use: formoterol, arformoterol and salmeterol. The long acting muscarinic receptor antagonist (LAMA) tiotropium is also available. These long-acting bronchodilators have a significantly positive effect on FEV1, lung volumes, dyspnea, exacerbation rate and quality of life ${ }^{12-16}$. Since inflammation in patients with COPD is, at least partly, corticosteroid-resistant, due to a reduced activity of the histone deacetylase the use of glucocorticosteroids (GCs) remains controversial ${ }^{10,12,16-21}$. However, this treatment is recommended for moderate/severe COPD rather than mild COPD since there is no other therapeutic option $^{10,13}$. A large clinical trial has shown improvements in lung function, and decreased exacerbation frequency in individuals receiving combined inhaled GC and LABA treatment versus placebo and oral GC therapy is also recommended for the treatment of COPD exacerbations $^{10-13,18-21}$.

The use of theophyllines has been associated with a reduction in the rate of COPD exacerbations compared with long-acting $\beta 2$-agonists among COPD patients. Theophyllines could be seen as an interesting alternative 
<smiles>CC(C)Cc1ccc([C@H](C)C(=O)NS(C)(=O)=O)cc1</smiles>

Reparixin $^{\mathrm{a}}$<smiles>C[C@H](CO)Nc1nc(SCc2cccc(F)c2F)nc2nc(N)sc12</smiles>

Compound $6^{\mathrm{b}}$<smiles>O=C(Nc1ccc(Cl)c(S(=O)(=O)N2CCNCC2)c1O)Nc1cccc(F)c1Cl</smiles>

SB656933

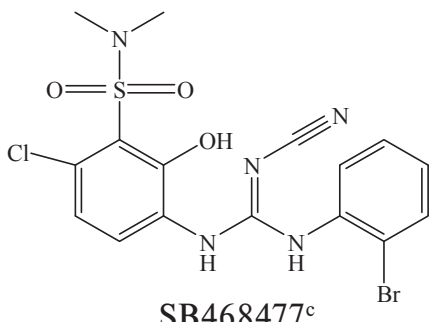

SB468477<smiles>CC(C(N)=O)c1ccc(OS(=O)(=O)C(F)(F)F)cc1</smiles>

DF2162<smiles>O=c1[nH]c2nc(SCc3cccc(Cl)c3F)nc(NCCO)c2s1</smiles>

AZ-10397767 ${ }^{\mathrm{b}}$<smiles>NS(=O)(=O)c1c(Cl)ccc(NC(=O)Nc2cccc(Cl)c2Cl)c1O</smiles>

SB332235<smiles>C=C(C(=O)NC)/C(O)=C(\C)Nc1c(N[C@H](CC)c2ccc(C)o2)c(=O)c1=O</smiles>

SCH527123

Fig. 2. Different inhibitors of chemokine receptors. CXCR2 antagonist report to be in development by pharmaceutical companies: ${ }^{\mathrm{a}}$ Dompe S.P.A. ${ }^{\mathrm{b}}$ Astra ${ }^{\mathrm{c}}$ GlaxoSmithKline and ${ }^{\mathrm{d}}$ Schering-Plough

in the treatment of COPD, because they are much less expensive than long-acting $\beta 2$-agonists, and, from the patient's perspective, an oral formulation might be easier to take than an inhaled formulation ${ }^{22}$.

At present, new drug developments in COPD focus on see (Fig.1) (ref. ${ }^{23}$ ) improved bronchodilators, novel anti-inflammatory drugs, antiproteases, and regenerative therapy strategies.

\section{New bronchodilators}

Although patients with COPD were initially considered to have fixed or irreversible airway obstruction, there is evidence that the airway obstruction is partially responsive to bronchodilators ${ }^{1-4}$. At present, LABAs are administered twice daily; nonetheless, in order to improve adherence, several once daily formulations of LABAs are in clinical development, the most promising are carmoterol and indacaterol ${ }^{19-21}$.
New $\beta$ 2-adrenoreceptor agonists

Carmoterol is a non-catechol $\beta$ 2-adrenoreceptor highly potent and selective for the $\beta 2$ receptor agonist ${ }^{23-26}$. The pharmacokinetics of carmoterol is proportional to the dose, with a linear accumulation of the drug after repeated dosing ${ }^{23-26}$. The compound, in combination with hydrofluoroalkane propellant, reaches a lung deposition of about $41 \%$ in controls and COPD patients. Carmeterol seems to provide a high therapeutic ratio since in clinical $2 \mu \mathrm{g}$ carmoterol once daily was as effective as $12 \mu \mathrm{g}$ formoterol twice daily with similar safety and tolerability ${ }^{23}$ however, due to the complex co-morbidities in COPD patients more studies are required to demonstrate safety and a minimum of adverse effects.

In animal studies, indacaterol had been shown to offer a similarly quick onset but a longer duration of action compared to salbutamol and formoterol ${ }^{23,24}$. Clinical trials evaluating the safety and efficacy of indacaterol in patients with COPD and asthma, demonstrated single 
doses of indacaterol provide a sustained bronchodilator effect over $24 \mathrm{~h}^{24}$ Indacaterol has shown a good safety profile in single daily doses from 50 to $600 \mu \mathrm{g}$ and no loss of efficacy after 7 days of treatment with effective 24 $\mathrm{h}$ bronchodilatation effect after at least $1 \mathrm{~h}$ post inhalation. Recently, in 635 patients with moderate to severe COPD, indacaterol demonstrated dose-dependent significant improvements of FEV1, similar to tiotropium, but with a faster onset of action ${ }^{23,24}$. Improvement of lung function persisted throughout a $24 \mathrm{~h}$ dosing interval, with a good safety profile over a 28-day period ${ }^{21}$. COPD exacerbations and symptoms, and health-related quality of life were also significantly improved for indacaterol versus placebo recipients in some studies. Indacaterol was generally well tolerated by adults with moderate to severe COPD. Indacaterol has been approved in Europe and the dosage 150 and $300 \mu \mathrm{g}$ for routine use.

In a recent review ${ }^{24}$ the short and long beta agonists in combination therapy were compared, the efficacy of formoterol and arformoterol are similar suggesting that new combination therapies may be considered. Several combinations, like budesonide/formoterol and beclomethasone/formoterol, have been successfully used in several trials with COPD patients and new combination therapies are underway to improve adrenergic receptor response decreasing possible side effects.

Several other long-acting $\beta$ 2-agonists are under development, including milveterol (GSK159797/TD3327) and GSK 642444. One such agent, GSK-159797, is a novel crystalline form of a formoterol derivative that showed good efficacy throughout a $24 \mathrm{~h}$ evaluation period and was well tolerated with no increase in heart rate. GSK 642444 potentially has a greater therapeutic index compared to GSK-159797. To date however, the available information about both compounds is limited ${ }^{23-26}$.

Combination inhalers with long-acting $\beta 2$-agonists (LABA) and long-acting muscarinic antagonist (LAMA) are also in development since an additive effect has been described $^{23-24}$. Moreover, single molecules that link a muscarinic antagonist to a $\beta 2$-agonist (MABA) are now also in development ${ }^{23,24}$.

\section{Acetylcholine receptor antagonists}

Aclidinium (LAS-34273) shows selectivity, long duration of action and rapid clearance from plasma ${ }^{23-26}$. Compared to tiotropium and ipratropium, aclidinium exhibits potent anticholinergic activity with a faster onset of action than tiotropium and a long duration ${ }^{23-26}$. Aclidinium improved specific airway conductance and increased FEV1 and forced vital capacity values, and it provided significant and sustained protection against metacholine-induced airway constriction over $24 \mathrm{~h}^{23-26}$. Significant bronchodilatation was induced by aclidinium after $15 \mathrm{~min}$ and sustained for at least $24 \mathrm{~h}$. Aclidinium was well tolerated as demonstrated in a Phase I study ${ }^{23-26}$.

The compound NVA 237 shows high affinity for the muscarinic receptors M1 and M3 with a slow disassociation constant from the $\mathrm{M} 3$ receptor providing a long duration of bronchodilator activity ${ }^{23-25}$. Inhaled NVA 237 has a low systemic adsorption suggesting that it may induce less systemic adverse effects ${ }^{23-25}$. A 5 min post dose onset of action had been demonstrated with a similar bronchodilatory effect as salbutamol with a longer half life. Further clinical studies are required to ascertain a sustained increase in FEV1 over long periods of time ${ }^{23-25}$.

The 4-acetamidopiperidine derivative OrM3 has about 120-fold selectivity for the M3 receptor over the M2 receptors $^{23-25}$. Interestingly, OrM3 is available as a tablet, being probably advantageous as a more convenient formulation than aerosol devices. OrM3 has a half-life of $>14 \mathrm{~h}$ thus, it may be used once-daily. OrM3 improved significantly dose related serial FEV1 and, in the Saint George questionnaire, a statistical trend was recorded when symptom relief scores were compared to placebo ${ }^{25}$. However, using doses that provide reduced efficacy compared with ipratropium, specific mechanism-related side effects exceeded those observed for ipratropium, including dry mouth, constipation, urinary retention and other disorders ${ }^{23-26}$.

CHF 5407 is an antagonist to M3 and M2 receptors with a potent and long lasting effect on the human M3 receptor. It shows a significantly shorter action at the M2 receptor and faster dissociation constant than tiotropium. When bronchospasm was induced by acetylcholine in guinea pigs, inhalation of CHF 5407 showed a two to three time's higher bronchodilatory activity than tiotropium and ipatropium. Further studies showed that aerolized CHF 5407 protects against acetylcholineinduced bronchospasm for at least $24 \mathrm{~h}^{23}$. Nonetheless, there are still important issues to address concerning pharmacokinetics and adverse effects.

Several researchers have proposed that pharmacogenetic studies are also required for these new bronchodilators as it may provide evidence of risk associated diseases that are often observed in COPD patients.

\section{Phosphodiesterase 4 inhibitors}

PDE4 is the predominant phosphodiesterase expressed in neutrophils, T-cells and macrophages, suggesting that PDE4 inhibitors would be effective in controlling inflammation in COPD. PDE4 inhibitors had been shown to have a broad anti-inflammatory capacity with a well documented efficacy in animal models of COPD. The effect depends largely on the blockage of chemokines synthesis and secretion ${ }^{26}$. Several oral PDE4 inhibitors are in clinical trials for COPD ${ }^{23-27}$. The main drawback is that the drugs are not selective for the PDE4D isoenzyme and cause side effects: nausea, emesis and headache ${ }^{23-27}$.

Several Phase II studies have assessed the efficacy of the PDE4 inhibitor cilomilast ${ }^{27}$. One placebo-controlled study of anti-inflammatory and physiologic efficacy showed no significant difference in FEV1 (ref. ${ }^{27}$ ). Despite the fact that CD4 and CD8 cells were significantly reduced in the airways, the drug had no effect on the number of invading neutrophils or on the transcription of IL- 8 or TNF- $\alpha$ in the inflamed tissue ${ }^{27}$. In a 24 -week period, cilomilast improved FEV1 baseline by $40 \mathrm{ml}$ as compared to placebo which was considered marginally significant in the Saint George respiratory questionnaire, and only $12 \%$ of the subjects in the cilomilast group were exacerbation free ${ }^{27}$. 
Roflumilast, another PDE4 inhibitor that prevents the breakdown of cyclic AMP, reduces the numbers of neutrophils (by 36\%) and CXCL8 concentrations in sputum of COPD patients treated over a 4-week period ${ }^{27-29}$. Roflumilast given over 6 or 12 months modestly improves lung function without decreasing exacerbations in COPD patients $^{28,29}$. In a Phase III, multi-center, double-blind, randomized, placebo controlled study, post bronchodilator FEV1 ( $p<0.0001)$ was significantly improved with roflumilast compared to placebo ${ }^{28,29}$. Roflumilast is currently licensed in Europe and it is registered in the Czech Republic as Daxas.

The limited efficacy of these PDE4 inhibitors is dependent upon the concentration required to achieve the maximum effect without adverse effects. Several pharmacological approaches have been designed: PDE4 inhaled inhibitors and PDE7 inhibitors ${ }^{27}$. The inhaled inhibitors were found to be ineffective, although well tolerated and the PDE7A inhibitor does not have sufficient antiinflammatory effects. However, early studies using the combination PDE7A and PDE4 inhibitor seem to potentiate the anti-inflammatory effects without increasing side effects ${ }^{27-29}$. Future studies are required to develop new formulations.

\section{Chemokine receptor antagonists}

In several studies, IL-8 was increased in the sputum of patients with COPD and correlated with disease severity and the risk of future exacerbations ${ }^{3,4}$. IL- 8 exerts its effects by binding to the chemokine receptors CXCR1 and CXCR2 on neutrophils ${ }^{30}$. An interesting possibility is to block CXCR2, which mediates the chemotactic effect of CXCL8 and CXCL1 on neutrophils and monocytes. There are two oral CXCR2 antagonists in clinical Phase I development: AZD8309 and SCH527123 (ref. ${ }^{21,23}$ ). ADZ8309 has recently been shown to inhibit neutrophil inflammation in the lung following inhaled endotoxin challenge in normal volunteers. Another possibility may be to block chemokine receptors CXCR3 since its ligands CXCL9, CXCL10 and CXCL11 levels are increased in COPD as well as the T cell CD $4+$ and CD $8+$ subpopulations that express increasing numbers of CXCR3 $\left(\right.$ ref. $^{23,30}$ ).

A number of pharmaceutical companies have successfully identified CXCR2 antagonists, several of which have been progressed into clinical trials. Listed below is a detailed description of the in vitro pharmacology of several CXCR2 antagonists. The chemical structures of these compounds are shown in (Fig. 2) $\left(\right.$ ref. $^{30}$ ).

A set of CXCR1/CXCR2 inhibitors developed by Dompé S.P.A. has advanced into clinical trials for the treatment of post-ischemia/reperfusion injury. The ketoprofen derivative, reparixin ( $R(-)$-2-(4-isobutylphenyl)propionyl methanesulfonamide) was characterized as an allosteric CXCR1-selective inhibitor of human PMN chemotaxis ${ }^{21,30}$. The compound is reported to inhibit CXCL8-mediated PMN migration with single-digit nanomolar potency while CXCR2-mediated PMN activation. The improved pharmacokinetic properties of these compounds have facilitated preclinical testing in more chronic diseases such as adjuvant-induced polyarthritis ulcerative colitis and prevention of tumor progression in melanoma. The clinical status of these compounds at this time is not known ${ }^{21,30}$.

A fused pyrimidine series-based CXCR2 antagonists has been developed by AstraZeneca, AZD-8309, for the treatment of rheumatoid arthritis and COPD ${ }^{23}$. The effect of AZD-8309 on lipopolysaccharide (LPS)-induced neutrophilic inflammation was assessed in healthy volunteers using a crossover study ${ }^{30}$. On the third day, following twice a day administration of AZD8309 or placebo, subjects were challenged with inhaled LPS and induced sputum was collected and processed for measurement of cells and mediators ${ }^{23}$. Treatment with AZD8309 resulted in a significant $(\sim 80 \%)$ reduction in total sputum cells and neutrophils compared with placebo after LPS challenge as well as a significant reduction in neutrophil elastase activity in the sputum and $\sim 40-50 \%$ reductions in the number of sputum macrophages and the levels of sputum LTB4 and CXCL8 $\left(\right.$ ref. ${ }^{30}$ ). The structure and pharmacology of AZD8309 have not been publicly disclosed, and its development was reportedly discontinued in February 2007. AstraZeneca recently published on structure-activity relationship studies of thiazolopyrimidine based CXCR2 antagonists. One such compound (Compound 6, Fig. 2) binds the receptor with reasonable affinity (functional $\mathrm{pA} 2 \sim 1 \mathrm{nM}$ ), although its rat oral bioavailability is relatively modest (9\%). There are indications that AstraZeneca may be developing bicyclic CXCR2/CCR2 dual antagonists which are also thiazolopyrimidine-based. One such compound, AZ-10397767 (Fig. 2) binds with single-digit nanomolar affinity to both receptors and has good oral bioavailability but relatively short clearance in rats ${ }^{30}$.

The cyanoguanidine compound, SB-468477 (N-(2hydroxy-3- dimethylsulfonylamido-4-chlorophenyl)-N'(2-bromophenyl)-N"-cyanoguanidine) (Fig. 2) inhibits chemokine binding to both CXCR1 and CXCR2 with binding IC50 values of $\sim 67$ and $12 \mathrm{nM}$, respectively ${ }^{30}$. In monocyte chemotaxis assays, $\sim 400$ nM SB- 468477 was sufficient to maximally inhibit cell migration to the CXCR2-selective ligands, CXCL1, CXCL5 and CXCL7. SB-656933 (Fig. 2) is a potent CXCR2 inhibitor (binding IC50=5.1 $\mathrm{nM}$ ) which was reported to inhibit CXCL1-stimulated neutrophil CD11b up regulation (IC50 $260 \mathrm{nM}$ ) and shape change (IC50 $310 \mathrm{nM}$ ) in whole blood samples from COPD patients. A clinical predecessor of SB-656933, SB-332235 (N-(2-hydroxy-3sulfamyl-4-chlorophenyl)-N'-(2,3dichlorophenyl) urea), also binds with selectivity for CXCR2 with similar potency to SB-656933 (binding IC50 5 nM). SB-332235 was reported to inhibit CXCL1- and CXCL8-stimulated CXCR2 activation (but not CXCR1) in human neutrophils. It also reduced CXCL1- induced neutrophil CD11b expression both in vitro and in vivo, and impaired recruitment of $\mathrm{CD} 11 \mathrm{~b}+$ neutrophils in the BAL fluid from aerosolized LPS-exposed rats $^{30}$. It is possible that new molecules with dual receptor inhibition with be introduced for clinical studies.

Recently, a potent CXCR1/CXCR2 antagonist, $\mathrm{SCH}$ 527123 [2-hydroxy-N,N,-dimethyl-3-[[2-[[1(R)-(5-methyl2-furanyl)propyl] amino]-3,4-dioxo-1-cyclobuten-1-yl]amino]benzamide] was described. SCH 527123 (Fig. 2) binds 
with nanomolar affinity for hCXCR1 $(\mathrm{Kd}=3.9 \pm 0.3 \mathrm{nM})$ and picomolar affinity for hCXCR2 $(\mathrm{Kd}=0.049 \pm 0.004$ $\mathrm{nM})^{30}$. Consistent with its high affinity, SCH 527123 binds CXCR2 with impressive avidity, dissociating with a t1/2 $22 \mathrm{~h}$ at ambient temperature. In functional assays, 3-10 nM SCH 527123 effectively ablated CXCL1stimulated chemotaxis of human PMN or recombinant $\mathrm{Ba} / \mathrm{F} 3-\mathrm{hCXCR} 2$ cells (i.e. CXCR2-mediated). In parallel, experiments examining CXCL8-stimulated chemotaxis of human polymorphonuclear cells or recombinant cells expressing CXCR1, a significant dextral displacement in the agonist concentration-response curves of cell migration were apparent only with high concentrations of SCH 527123 (200-300 nM). Therefore, although both the cyanoguanidine compounds SB-468477 and SCH 527123 have activity at both CXCR1 and CXCR2, both compounds are effectively CXCR2- selective. SCH 527123 has demonstrated efficacy in a variety of pulmonary inflammation models. In a clinical study, this compound inhibited ozone inhalation-induced sputum neutrophil recruitment ${ }^{30}$.

\section{NFkB inhibition}

The transcription factor NF-kB regulates the expression of chemokines, inflammatory cytokines and proteases, including IL-8, TNF- $\alpha$ and MMP-9. NF- $\kappa B$ is activated in macrophages and epithelial cells of COPD patients, particularly during exacerbations ${ }^{1,23,25,31}$.

It has been shown that overexpression of IKK- $\beta$ in mouse airway epithelial cells results in an increase in inflammatory mediators and neutrophilic inflammation that is reminiscent of the COPD airway following bacterial challenge ${ }^{31}$. In addition, inhibition of IKK- $\beta$ in vivo and in vitro reduced TNF- $\alpha$ induced MUC5AC production, one of the major components of respiratory mucus ${ }^{10,11}$. Production of another important respiratory mucin MUC5B has also been shown to be IKK- $\beta$ dependent, following rhinovirus infection in vitro. Transfection of alveolar macrophages with adenovirus constructs expressing defective IKK- $\beta$ but not NIK proteins inhibited macrophage activation of NF-kB, and expression of TNF- $\alpha$, IL-8/CXCL8 and IL-6. Monocyte derived macrophages infected in vitro with RV produce TNF- $\alpha$ in an NF-kB dependent manner, which is sensitive to treatment with the IKK- $\beta$ inhibitor AS206828 (ref. ${ }^{31}$ ).One inhibitor of NF-kB kinase (IKK) 2 was tested on animal models of $\mathrm{COPD}^{31}$. It showed efficiency in lipopolysaccharide exposed animals, but not in elastase-induced emphysema. Additionally, IKK2 inhibitors depress CXCR3 chemokines, suggesting potential complex interactions between signal transduction pathways. Although several IKK2 inhibitors are now in development, so far none has been tested in COPD patients ${ }^{31}$.

\section{Prophylactic antibiotics}

It is at present believed that most exacerbations are triggered by viral and bacterial infections. With this as a background, the issue of chronic bacterial colonization/ infection and long-term antibiotic therapy gained attention. A meta-analysis of older randomized trials that studied the prophylactic effect of antibiotics indicated that antibiotics significantly decreased the likelihood of having an exacerbation ${ }^{32}$. However, the relative reduction was only $9 \%$ and there was no relevant effect on loss of lung function ${ }^{21,24,32}$.

In the past decades, evidence suggested that there are beneficial effects of erythromycin in patients with COPD not only mediated by conventional antibacterial effects, but rather by a variety of anti-inflammatory and immunomodulatory actions. A recent single-center, randomized controlled trial investigated prophylactic erythromycin (250 mg, twice daily) versus placebo for 1 year in 109 patients with moderate to severe COPD showed that the macrolide reduce exacerbation frequency in relative terms by $35 \%$ compared to placebo ${ }^{32}$. Actually, the National Heart, Lung and Blood Institute is conducting a multi-center randomized clinical trial of 1130 patients with COPD at high risk for exacerbations in which participants receive either daily azithromycin $(250 \mathrm{mg})$ or placebo for 1 year with the primary outcome time to first exacerbation. The PULSE trail showed that intermittent pulsed antimicrobial therapy with moxifloxacin provided a significant reduction in the frequency of exacerbations in patients with COPD. However, it should be taken into account that macrolide therapy for reduction of exacerbations in patients at high risk for exacerbation may include millions or even tens of millions of COPD patients on a global scale. This may result in a widespread emergence of macrolide bacterial resistance ${ }^{23-26,32}$.

\section{Lipid antagonists}

Leukotriene (LT) B4 is increased in sputum and bronchoalveolar lavage fluid of patients with COPD and is chemotactic for neutrophils and lymphocytes ${ }^{3,16-19}$. Several antagonists of the major receptor BLT1 have developed, but so far clinical studies in COPD have been negative. 5-Lipoxygenase inhibitors should also be beneficial by blocking the production of endogenous LTB4, but it has been difficult to develop potent safe 5-LO inhibitors ${ }^{21,22}$.

\section{Cytokine inhibitors}

The front runner amongst the mediators of COPD must be tumor necrosis factor- $\alpha$ (TNF- $\alpha$ ), as its concentrations are increased in COPD. It amplifies inflammation and may account not only for neutrophilic inflammation in the lungs but also some systemic features such as skeletal muscle wasting ${ }^{21,33,34}$. However, blockade of TNF- $\alpha$ with an injected antibody (infliximab) had no beneficial clinical effects in patients with COPD, using the same doses which are effective in rheumatoid arthritis and which have shown a beneficial effect in asthma ${ }^{33}$. Of particular concern was the finding that more COPD patients treated with anti-TNF $\alpha$ developed cancers of the respiratory tract and severe lung infections. This may have implications for other treatments discussed later that inhibit TNF- $\alpha$ production ${ }^{33}$. Other cytokines that are currently target for inhibition include IL-1 $\beta$, IL-6 and IL-17. IL-6 is increased in sputum and in the systemic circulation of COPD patients and may account for the increase in circulating C-reactive protein ${ }^{13,32,33}$. A potent inhibitor of 
IL-6 is the receptor antibody tocilizumab, which is effective in rheumatoid arthritis but has not yet been tested in COPD patients ${ }^{13,32,33}$.

New therapeutic approaches blocking IL-17 and Th17 generation seem to be useful in COPD essentially because IL-17 is involved in neutrophil recruitment and, it is crucial in the immune response to infections ${ }^{35}$.

\section{Antiproteases}

There is compelling evidence for an imbalance between proteases and antiproteases in COPD. This suggests that either inhibiting proteolytic enzymes or increasing the levels of antiproteases may be beneficial and theoretically should prevent the progression of emphysema. Neutrophil elastase (NE)-knockout mice are partially protected from developing emphysema in tobacco smoke models $^{36}$. A small molecule inhibitor of NE, ZD0892, was tested in animal models and reduced tobacco smokeinduced emphysema ${ }^{36}$. At present, there are nebulized inhibitors of NE in clinical development, but so far all have failed in clinical trials ${ }^{18,19}$.

The selective MMP-12 inhibitor AS111793 was tested in smoking mice and reduced the inflammatory process associated with exposure of mice to cigarette smoke ${ }^{36}$. A murine model of COPD demonstrated the use of inhaled AAT to reduce emphysema severity, lending more credence to this therapeutic approach. Further work in this area is indicated to clarify any potential benefit for patients $^{21,23,36}$.

There is compelling evidence for an imbalance between proteases that digest elastin (and other structural proteins) and antiproteases that protect against degradation. This suggests that either inhibiting these proteolytic enzymes or increasing endogenous antiproteases may be beneficial and theoretically should prevent the progression of emphysema. The fact that there are so many proteinases implicated in COPD might mean that blocking a single enzyme may not have a major effect endogenous antiproteases ( $\alpha 1$-antitrypsin, secretory leukoprotease inhibitor, elafin, tissue inhibitors of MMP), either in recombinant form or by viral vector gene delivery. These approaches are unlikely to be cost effective as large amounts of protein have to be delivered and gene therapy is unlikely to provide sufficient protein ${ }^{36}$. A more promising approach is to develop small molecule inhibitors of proteases, particularly those that have elastolytic activity. Neutrophil elastase inhibitors have been developed but have all failed in clinical trials. Matrix metalloproteinases (MMPs) with elastolytic activity are also a target for drug development, and MMP-9 appears to be the predominant enzyme, which is released from macrophages, neutrophils and epithelial cells ${ }^{36}$. Non-selective MMP inhibitors, such as marimastat, appear to have major side effects, suggesting that isoenzyme selective inhibitors or inhaled delivery may be needed ${ }^{36}$. A dual MMP-9/MMP-12 inhibitor, AZ11557272, has been shown to prevent emphysema and small airway thickening in guinea pigs exposed to cigarette smoke over six months, but the clinical development of this inhibitor has been stopped. ${ }^{36}$

\section{Retinoic acid}

Retinoic acid increases alveolar septation during lung development. Animal experiments suggested that all- trans -retinoic acid induces regeneration of the terminal respiratory tract as it was shown to reverse elastase-induced emphysema. However, several other studies could not confirm this observation. A clinical trial of all-trans -retinoic and 9- $c i s$ - retinoic acid in patients with emphysema failed to show any improvement in clinical parameters, health status or CT density after 6 months of therapy ${ }^{21,26,35}$.

\section{Regenerative therapies}

Another possible approach for regeneration of destroyed lung tissue in emphysema is the use of stem cells to seed in the lung. In combination with drugs that stimulate their homing and proliferation in the lung, they will potentially provide regenerative capacity. Human embryonic stem cells have already been transformed into alveolar type II pneumocytes that might have the capacity to repair alveolar damage. However, there is still a long way to go, particularly because the lung is a complex organ that probably needs to grow both endothelial and alveolar cells to repair emphysema ${ }^{21,23,37,38}$.

\section{CONCLUSIONS}

Several pharmacological approaches have been used for the treatment of COPD; however, it has not been an easy task, new bronchodilators, and new anti inflammatory anti migration therapies have been used without major improvement. However, the late onset of the disease is the main problem to solve in these new therapies and schemes. Hopefully new drug combinations may result in more effective in decrease in disease progression and exacerbations in this complicated disease. Further studies are also required to understand the molecular epigenetic and pharmacology of this disease, one of the commonest diseases in the elderly.

\section{ACKNOWLEDGEMENTS}

Financed by a project by the Venezuelan National Foundation of Research (FONACIT), number G-2005000389, by the Consejo de Desarrollo Científico y Humanístico Universidad Central de Venezuela, project \# $P G$ 09.00.6785.07 and by the Research Coordination of the Faculty of Medicine.

\section{REFERENCES}

1. Global Strategy for Diagnosis, Management and Prevention of COPD Updated 2009. Global Initiative for Chronic Obstructive Lung Disease. http://www.goldcopd.org.

2. Cosio MG, Saetta M, Agusti A. Immunologic Aspects of Chronic Obstructive Pulmonary Disease. N Engl J Med 2009;360:2445-54.

3. Barnes PJ. Immunology of asthma and chronic obstructive pulmonary disease. Nat Rev Immunol 2008;8(3):183-92.

4. Curtis JL, Freeman CM, Hogg JC. The Immunopathogenesis of Chronic Obstructive Pulmonary Disease. Insights from Recent Research. Proc Am Thorac Soc 2007;4:512-21. 
5. Rennard SI. Chronic Obstructive Pulmonary Disease Linking Outcomes and Pathobiology of Disease Modification. Proc Am Thorac Soc 2006;3:276-280.

6. Anto JM, Vermeire P, Vestbo J, Sunyer J. Epidemiology of chronic obstructive pulmonary disease. Eur Respir J 2001;17:982-94.

7. American Thoracic Society. Standards for the diagnosis and care of patients with chronic obstructive pulmonary disease. Am J Respir Crit Care Med 1995;152:S77-S121.

8. Pauwels RA, Buist AS, Calverley PM, Jenkins CR, Hurd SS, the GOLD Scientific Committee. Global strategy for the diagnosis, management, and prevention of chronic obstructive pulmonary disease. NHLBI/WHO Global Initiative for Chronic Obstructive Lung Disease (GOLD) Workshop summary. Am J Respir Crit Care Med 2001;163:1256-1276.

9. Celli BR, MacNee W. Standards for the diagnosis and treatment of patients with COPD: a summary of the ATS/ERS position paper. Eur Respir J 2004;23:932-46.

10. Barnes PJ. Targeting the epigenome in the treatment of asthma and chronic obstructive pulmonary disease. Proc Am Thorac Soc 2009;6(8):693-6.

11. Cavalcante AG, de Bruin PF. The role of oxidative stress in COPD: current concepts and perspectives. J Bras Pneumol 2009;35(12):1227-37.

12. Barnes PJ, Stockley RA. COPD: current therapeutic interventions and future approaches. Eur Respir J 2005;25:1084-1106.

13. Barnes PJ. New therapies for chronic obstructive pulmonary disease. Med Princ Pract 2010;19(5):330-8.

14. Buhl R, Farmer SG. Future Directions in the Pharmacologic Therapy of Chronic Obstructive Pulmonary Disease. Proc Am Thorac Soc 2005;2:83-93.

15. Celli B, Goldstein R, Jardim J, Knobil K. Future perspectives in COPD. Respiratory Medicine 2005;99:S41-S48.

16. Hanani NA, Ambrosino N, Calverley P, Cazzola M, Donner CF, Make B. Treatments for COPD. Respiratory Medicine 2006;99:S28-S40.

17. Kolb M, Martin G, Medina M, Ask K, Gauldie J. Gene Therapy for Pulmonary Diseases. Chest 2006;130:879-884.

18. Meinke L, Chitkara R, Krishna G. Advances in the management of chronic obstructive pulmonary disease. Expert Opin Pharmacother 2007:8(1):23-37.

19. Montuschi P. Pharmacological treatment of chronic obstructive pulmonary disease. Internacional Journal of COPD 2006;1(4):409423.

20. Pelaia G, Vatrella A, Gallelli L, Renda T, Caputi M, Maselli R, Marsico SA. Biological targets for therapeutic interventions in COPD: clinical potential. Int J Chron Obstruct Pulmon Dis 2006;1(3):321-334.

21. Seifart C, Vogelmeier C. Emerging drugs in chronic obstructive pulmonary disease. Expert Opin Emerging Drugs 2009;14(1):181-194.

22. Cyr MC, Bauchesne MF, Lemiere C, Blais L. Effect of theophylline on the rate of moderate to severe exacerbations among pa- tients with chronic obstructive lung disease. Br. J Clin Pharmacol $65(1), 45-50$

23. Onoue S, Misaka S, Kwabata Y, Yamada S. New treatments for chronic obstructive pulmonary disease and viable formulation/ device options for inhalation therapy. Expert Opin Drug Deliv 2009;6(8):793-81.

24. Tashkin DP, Fabbri LM. Long-acting beta-agonists in the management of chronic obstructive pulmonary disease: current and future agents. Respir Res 2010;11:149.

25. Barnes PJ. Frontrunners in novel pharmacotherapy of COPD. Current Opinion in Pharmacology 2008;8:300-307.

26. Cazzola M, Matera MG. Novel long-acting bronchodilators for COPD and asthma. British Journal of Pharmacology 2008;155:291-299.

27. Currie GP, Butler CA, Anderson WJ, Skinner C. Phosphodiesterase 4 inhibitors in chronic obstructive pulmonary disease: a new approach to oral treatment. Br J Clin Pharmacol 2008;65(6):803-810.

28. Giembycz MA, Field SK. Roflumilast: first phosphodiesterase 4 inhibitor approved for treatment of COPD. Drug Des Devel Ther 2010;4:147-58.

29. Gross NJ, Giembycz MA, Rennard SI. Treatment of chronic obstructive pulmonary disease with roflumilast, a new phosphodiesterase 4 inhibitor. COPD 2010;7(2):141-53.

30. Chapman RW, Phillips JE, Hipkin RW, Curran AK, Lundell D, Fine JS. CXCR2 antagonists for the treatment of pulmonary disease. Pharmacology \& Therapeutics 2009;121:55-68.

31. Edwards MR, Bartlett NW, Clarke D, Birrell M, Belvisi M, Johnston SL. Targeting the NF-kB pathway in asthma and chronic obstructive pulmonary disease. Pharmacology \& Therapeutics 2009; $121: 1-13$.

32. Friedlander AL, Albert RK. Chronic macrolide therapy in inflammatory airways diseases. Chest 2010;138(5):1202-12.

33. Matera MG, Calceta L, Cazzola M. TNF-a inhibitors in asthma and COPD: We must not throw the baby out with the bath water. Pulmonary Pharmacology \& Therapeutics 2010;23:121-128.

34. Marwick JA, Chung KF, Adcock IM. Phosphatidylinositol 3-kinase isoforms as targets in respiratory disease. Ther Adv Respir Dis 2010;4(1):19-34.

35. De Sanctis JB, Garmendia JV, Moreno D, Larocca N, Mijares M, Di Giulio C, Salazar-Bookaman M, Radzioch D. Pharmacological modulation of Th17. Rencent Patents in Allergy and Immunology 2009;3(2):149-56

36. Chen ZH, Kim HP, Ryter SW, MK Choi A. Identifying targets for COPD treatment through gene expression analyses. International Journal of COPD 2008;3(3):359-370.

37. Wong S, Belvisi MG, Birrell MA. MMP/TIMP expression profiles in distinct lung disease models: implications for possible future therapies. Respir Res 2009;10:72.

38. Séguin RM, Ferrari N. Emerging oligonucleotide therapies for asthma and chronic obstructive pulmonary disease. Expert Opin Investig Drugs 2009;18(10):1505-1517. 